Ocular Oncology

and Pathology
Ocul Oncol Pathol 2016;2:156-159

DOI: $10.1159 / 000442987$
Received: September 24, 2015

Accepted after revision: December 1, 2015

Published online: December 19, 2015

\title{
Eyelid Metastatic Thyroid Papillary Carcinoma
}

\author{
Hardeep Singh Mudhar ${ }^{a}$ Murtuza Nuruddin ${ }^{b}$ Soma Rani Roy ${ }^{b}$ \\ a National Specialist Ophthalmic Pathology Service (NSOPS), Department of Histopathology, E-Floor, Royal

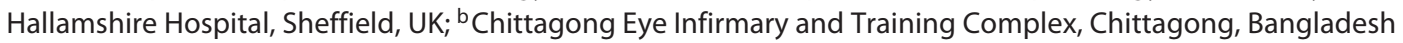

\section{Key Words}

Metastasis · Eyelid · Thyroid carcinoma · Papillary carcinoma

\begin{abstract}
Purpose: The aim of this study was to report the clinical and histopathological features of an eyelid metastasis in a 70-year-old male with no prior medical history. Methods: A 70-year-male presented with a painless, hard, red nodular growth in his left lower medial eyelid of 6 months duration, involving the canaliculus and punctum. He did not complain of any other ocular or systemic abnormality. The clinical differential diagnosis included lymphoma or a primary adnexal tumour. The lesion was excised under local anaesthetic cover and sent for routine histopathological analysis. Results: The histology revealed a carcinoma involving the posterior and anterior lamella of the lower lid. It was composed of papillary and follicular areas. The tumour nuclei were optically clear, overlapping, had nuclear grooves and were associated with eosinophilic colloid material. The tumour cells were strongly positive for nuclear thyroid transcription factor-1 and thyroglobulin (which also stained some of the eosinophilic colloid material). The histological and immunohistochemical features were those of metastatic papillary carcinoma of the thyroid. Unfortunately, the patient's thyroid has yet to be examined because of loss to follow-up. Conclusion:
\end{abstract}

To the best of our knowledge, this is the first report of thyroid papillary carcinoma metastasizing to the eyelid as the presenting feature of the disease. A review of the literature of metastatic thyroid carcinoma to the eyelids is presented, to place the findings of this case report in context.

C 2015 S. Karger AG, Basel

\section{Introduction}

The eyelid is composed of various tissues which can give rise to a wide range of benign and malignant primary neoplasms. Eyelid metastases represent $<1 \%$ of all lid malignancies [1-4]. In a series of 892 eyelid lesions, Aurora and Blodi [1] reported only 3 cases of metastases. In a larger series reported by Arnold et al. [2], there was only 1 metastasis out of 1,502 eyelid lesions examined. The AFIP series reported by Mansour and Hidayat [5] examined 31 metastatic eyelid tumours and found that the breast was the commonest primary location, followed by the skin, gastrointestinal tract and genitourinary tract. A smaller series of 15 cases reported by Riley et al. [6] reported a similar primary origin. We report on the clinical and histological features of a unilateral eyelid metastasis in a 70-year-old male, thought to be either lymphoma or a primary adnexal tumour on clinical grounds.

\section{KARGER}

E-Mail karger@karger.com

www.karger.com/oop
(C) 2015 S. Karger AG, Base

2296-4681/15/0023-0156\$39.50/0
Dr. Hardeep Singh Mudhar

National Specialist Ophthalmic Pathology Service (NSOPS)

Department of Histopathology, Royal Hallamshire Hospital

Sheffield S10 2JF (UK)

E-Mail hardeep.mudhar@sth.nhs.uk 

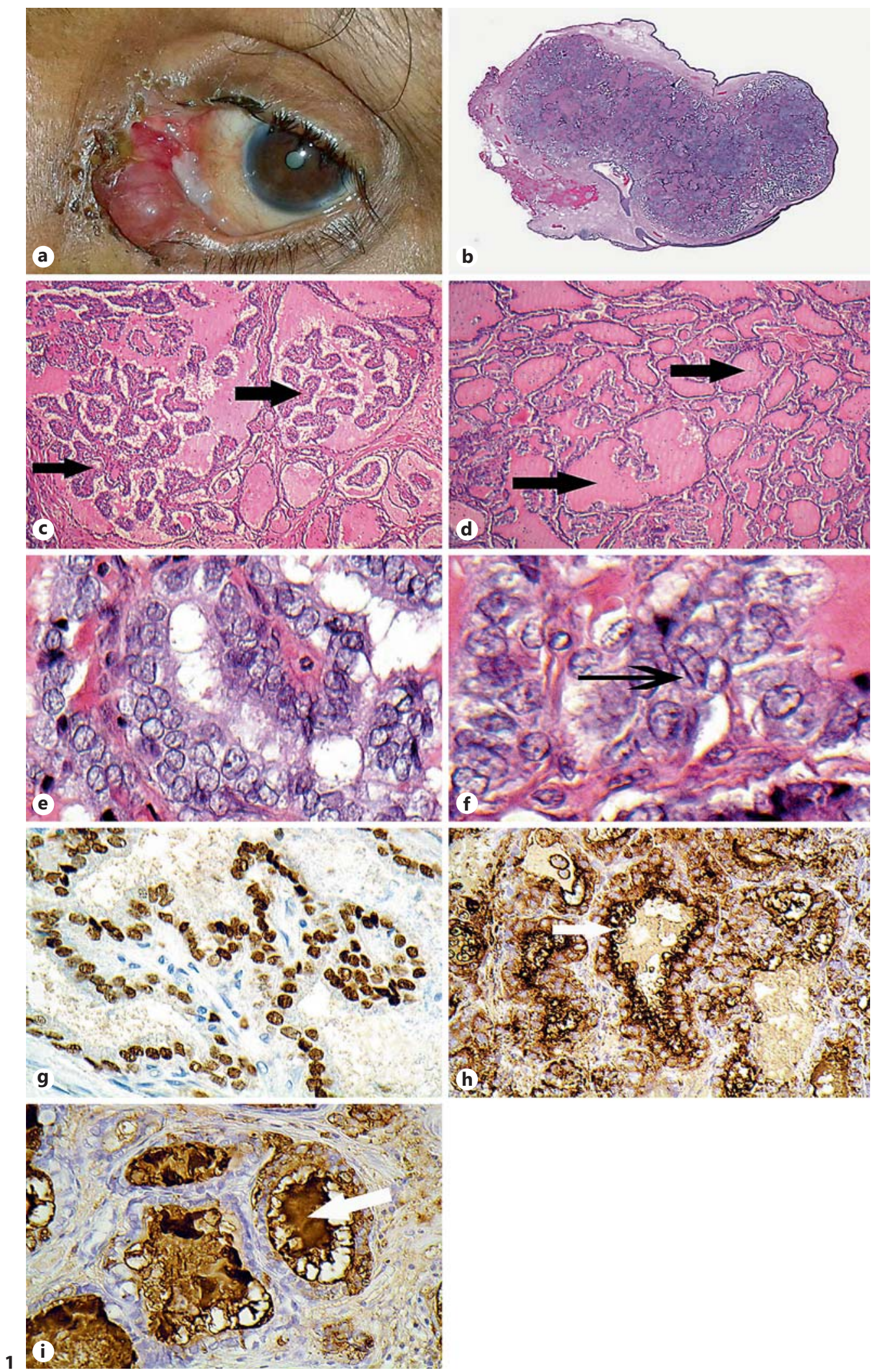

(For legend see next page.)

Eyelid Metastatic Thyroid Papillary Carcinoma
Ocul Oncol Pathol 2016;2:156-159 DOI: 10.1159/000442987 


\section{Case Report}

A-70-year-old Bangladeshi male patient attended the Orbit and Oculoplastic Clinic at The Chittagong Eye Infirmary with a complaint of a left-lower-lid lump of 6 months duration. On examination, a firm, non-tender lesion was situated at the medial end of the lower lid, involving the lower punctum and canaliculus. It was purplish and involved both the anterior and posterior lamella (fig. 1a). There was no proptosis, and no regional lymph nodes were palpable. His corrected visual acuity was 20/80 in both eyes due to senile immature cataract. The anterior segment was otherwise normal, as was the fundus, in both eyes. The patient had no significant past medical history. The lesion was excised under local anaesthesia. Tissue was fixed in standard buffered formalin and submitted for specialist ophthalmic pathology reporting.

The ophthalmic histopathology laboratory received a left-lower-lid full-thickness excision, measuring $25 \mathrm{~mm}$ side to side by 18 $\mathrm{mm}$ superior-inferior by $12 \mathrm{~mm}$ thick, bearing a smooth, reddish nodule $13 \times 8 \times 7 \mathrm{~mm}$ at the lid margin. Microscopy showed a tumour involving the anterior and posterior lamella of the eyelid (fig. 1b). At scanning power, it was composed of papillary (fig. 1c) and follicular areas (fig. 1d) with some eosinophilic colloid material. At higher power, the tumor nuclei were ovoid to circular with overlapping, optically clear nuclei with nuclear-edge accentuation (fig. 1e). Some nuclei showed grooves and contained nucleoli (fig. 1f). Occasional mitotic activity was identified. The colloid material had a bubbly appearance, especially where it was in contact with the epithelial cells. Immunohistochemistry showed the tumour cells to express nuclear thyroid transcription factor-1 (TTF1; fig. 1g) and cytoplasmic thyroglobulin (fig. 1h). In some areas, the colloid stained with thyroglobulin as well (fig. 1i). The histological and immunohistochemical appearances were those of unequivocal metastatic papillary carcinoma of the thyroid. The patient was contacted for a thyroid review but has been lost to follow-up.

\section{Discussion}

Metastases to the eyelids in known cases of malignancy are a rare finding [1-4]. Even rarer is an eyelid metastasis being the presenting feature of metastatic disease. This case described metastatic papillary thyroid carcinoma to the right lower eyelid. There have been many case reports documenting the different histological subtypes of thyroid carcinoma metastasizing to various ocular niches, such as the orbit and uveal tract (too

Fig. 1. a Reddish nodular lesion at the medial aspect of the left lower eyelid, involving the punctum and canaliculus. b Scanning power image of the histological section (HE). c Higher-power image, showing papillary areas of the tumour (HE; black arrows). d Another part of the tumour, showing follicular areas with distinct eosinophilic colloid material (HE; black arrows). e Higher- numerous to mention). However, a careful review of the literature has revealed only 1 previous eyelid case of metastatic thyroid carcinoma. The patient was a 56-yearold male with known metastatic medullary carcinoma (not associated with multiple endocrine neoplasia) with deposits in the supraclavicular and cervical lymph nodes and lungs who developed an upper right eyelid metastasis presenting 36 years after thyroidectomy. The eyelid deposit was associated with bilateral choroid metastases [7].

In our case, the nodule represented the first presenting sign of metastatic papillary thyroid carcinoma. The patient had no other ocular manifestations. However, it is unknown whether he had other sites affected by synchronous metastases as the patient did not attend the clinic to investigate the thyroid and for wider metastatic disease. In previous series of eyelid metastases, the eyelid lesion was the presenting feature of the tumour in 7\% [6], $45 \%$ [5] and 15\% [8] of the cases. Eyelid metastases can present in a number of ways. They may be solitary or multiple, of different hues, with or without ulceration [8]. By far the commonest eyelid metastasis in most case series is breast carcinoma [5-6].

This case illustrates the importance of excising and carefully examining the histology of eyelid lesions. The clinical differential diagnosis was thought to be either a primary adnexal malignancy or lymphoma. These latter diagnoses imply a very different management strategy compared to a metastasis. Furthermore, the important role of immunohistochemistry is illustrated by the use of antibodies, such as TTF-1 and thyroglobulin, to confirm the organ of origin of the metastasis. The microscopic distinction between lymphoma and thyroid papillary carcinoma is straightforward on morphology alone. There are only a few primary skin adnexal lesions that may be confused with metastatic thyroid papillary carcinoma, such as papillary eccrine adenoma and digital papillary carcinoma. However, the site, careful attention to the highly distinctive nuclear features (clearing and grooving), and the presence of colloid-like material, coupled with the immunohistochemical profile, would permit an accurate interpretation.

power image, illustrating the nuclear features of the tumour (HE). f Higher-power image, showing the nuclear grooves (HE; black arrow). $\mathbf{g}$ Nuclear TTF-1 immunohistochemical positivity of the tumour. $\mathbf{h}$ Cytoplasmic tumour-cell positivity with antibody to thyroglobulin (white arrow). i Colloid staining positively with thyroglobulin (white arrow). 


\section{Statement of Ethics}

The study was conducted in accordance with the Declaration of Helsinki.

\section{Disclosure Statement}

The authors declare that there are no conflicts of interest.

\section{References}

1 Aurora AL, Blodi FC: Lesions of the eyelids: a clinic pathological study. Surv Ophthalmol 1970;15:94-104.

2 Arnold AC, Bullock JD, Foos RY: Metastatic eyelid carcinoma. Ophthalmology 1985;92: 114-119.

3 Wang JK, Liao SL, Jou JR, et al: Malignant eyelid tumours in Taiwan. Eye 2003;17:216-220.
4 Weiner JM, Henderson PN, Roche J: Metastatic eyelid carcinoma. Am J Ophthalmol 1986;101:252-254.

5 Mansour AM, Hidayat AA: Metastatic eyelid disease. Ophthalmology 1987;94:667-670.

6 Riley FC: Metastatic tumors of the eyelids. Am J Ophthalmol 1970;69:259-264.
7 Bianciotto CG, Demirci HY, Shields CL, Shields JA. Simultaneous eyelid and choroidal metastasis 36 years after diagnosis of medullary thyroid carcinoma. Ophthal Plast Reconstr Surg 2008;24:62-63.

8 Bianciotto C, Demirci H, Shields CL, Eagle RC, Shields JA: Metastatic tumours to the eyelid report of 20 cases and review of the literature. Arch Ophthalmol 2009;127:999-1005. 